\title{
Performance bounds in terms of QoS parameters in LTE mobile wireless system
}

\author{
Samridhi $^{1}$, Dr. Jyoteesh Malhotra ${ }^{2}$ \\ Student, ECE Department, Guru Nanak Dev University Regional campus Jalandhar, India ${ }^{1}$ \\ Associate Professor, ECE / CSE Department, Guru Nanak Dev University Regional campus Jalandhar, India ${ }^{2}$
}

\begin{abstract}
A new technology which we call as Long term evolution (LTE) has brought blazingly fast services to our mobile equipment. LTE, considered as representative of fourth generation wireless systems or the next generation wireless communication, is developed by $3 \mathrm{GPP}$ and is specified in release- 8 to fulfill the necessities of this era. As LTE inherits a lot from previous 3GPP standards, it is simply the organic evolution of 3G predecessors.LTE has mainly four enabling technologies i.e. OFDM (Orthogonal Frequency Division Multiplexing), MIMO (Multiple Input Multiple Output), Link Adaptation and Turbo Coding. These technologies together contribute and help LTE to meet its requirements and accomplish its goals including high peak data rates, low latency, flexibility in bandwidth, improved capacity, better coverage, multi-antenna support, seamless integration with other open standards and so on. This paper represents a survey on performance bounds in terms of QoS parameters of these four technologies. Different parameters have been considered with respect to different techniques. On the basis of survey bounds that ultimately become bottleneck have been mentioned.
\end{abstract}

Keywords: LTE, Turbo coding, MIMO, OFDM.

\section{INTRODUCTION}

Long Term Evolution commonly referred to as $4^{\text {th }}$ transmission. Upper bounds on these four parameters generation of mobile broadband system has been decide the extent to which performance of LTE systems developed to enhance earlier standards with integration of can be expected. The rest of the paper has been designed new functionalities like link adaptation, intensified use of as follows: next section covers an overview about enabling multi-antenna technologies etc. On one hand it represents technologies and requirements of LTE.Third section disruptive departure from the past and on the other hand it consists of literature survey of upper bounds of is the dawn of what is to come. It features all-IP packet- performance in LTE mobile communication systems. It is based networks. Explosive demand for bandwidth-hungry followed by issues in next section. In the last section, open applications is one of the mandates of LTE. The IMT- issues and future scope has been given.

Advanced guidelines provide spectrum flexibility which leads to scalability in the frequency domain in LTE. It can utilize frequency bands of previous 3GPP standards as well as few new frequency bands have been introduced in its specification. LTE has a list of spectrum allocations ranging from 1.4 to $20 \mathrm{MHz}$.

To integrate all its requirements and functionalities, whether of tremendous data rates, marvelous range and capacity or ability to confront as well as avoid deformations, it utilizes applications of different techniques. Usage of turbo coding is an optimal way of boosting performance of LTE systems. It's the only channel coding mechanism for processing user's data.Another key technology deployed in LTE standard is MIMO. It boosts the data rate, increases average cell throughput and hence reliability of communication links.The basic transmission schemes i.e. OFDM (downlink) and its single-carrier counterpart SC-FDM (uplink) are used in LTE. They provide robustness to multipath fading channel, interference coordination and apply frequency selective scheduling. Next LTE link well as capability of the system i.e. whether it is SISO, interference and noise ratio. This parameter is used for tabulated as:

selecting modulation and coding scheme (MCS) for

\section{ENABLING TECHNOLOGIES AND REQUIREMENTS OF LTE}

The evolution of $3 \mathrm{G}$ into $4 \mathrm{G}$ is driven by formation and growth of new services for mobile devices. It is accredited by advancement of technology available for mobile systems. The major challenge was becoming capable to support the equal Internet Protocol (IP) based services in a mobile device that is used by people domestically with a fixed broadband connection and it became a key driver for the evolution of LTE.

It has capability of providing peak data rate up-to $300 \mathrm{Mbps}$ for downlink and $75 \mathrm{Mbps}$ for uplink, that too with latency less than $10 \mathrm{~ms}$.It supports six different channel bandwidths like 1.4, 3, 5, 10, 15 and 20MHz. with respect to each bandwidth there are different number of resource blocks respectively.

LTE capacity depends upon three main factors channel bandwidth, network loading and lastly on configuration as

coding scheme (MCS) for 
Table1. Resource Blocks, FFT and Cyclic Prefix (CP) sizes for different LTE bandwidths

\begin{tabular}{|c|c|c|c|c|c|c|}
\hline $\begin{array}{l}\text { Bandwidt } \\
\mathrm{h} \\
(\mathrm{MHz})\end{array}$ & 1.4 & 3 & 5 & 10 & 15 & 20 \\
\hline $\begin{array}{l}\text { Sampling } \\
\text { Frequency } \\
(\mathrm{MHz})\end{array}$ & $\begin{array}{l}1.9 \\
2\end{array}$ & $\begin{array}{l}3.8 \\
4\end{array}$ & $\begin{array}{l}7.6 \\
8\end{array}$ & $\begin{array}{l}15.3 \\
6\end{array}$ & $\begin{array}{l}23.0 \\
4\end{array}$ & $\begin{array}{l}30.7 \\
2\end{array}$ \\
\hline FFT Size & 128 & 256 & 512 & 1024 & 1536 & 2048 \\
\hline $\begin{array}{l}\text { Number } \\
\text { of } \\
\text { resource } \\
\text { blocks }\end{array}$ & 6 & 15 & 25 & 50 & 75 & 100 \\
\hline $\begin{array}{l}\text { OFDM } \\
\text { symbols } \\
\text { per slot }\end{array}$ & \multicolumn{4}{|c|}{$14 / 12$} & \multicolumn{2}{|c|}{$\begin{array}{l}\text { (Normal/ } \\
\text { extended) }\end{array}$} \\
\hline CP length & \multicolumn{4}{|c|}{$4.7 / 5.6$} & \multicolumn{2}{|c|}{$\begin{array}{l}\text { (Normal/ } \\
\text { extended) }\end{array}$} \\
\hline $\begin{array}{l}\text { OFDM } \\
\text { symbols/ } \\
\text { sub-frame }\end{array}$ & \multicolumn{6}{|c|}{ 7/6(short/long CP) } \\
\hline
\end{tabular}

In order to accommodate above requirements and hence to gain excellent data rates, better coverage and to reach other goals, LTE implements four technologies which are as:

Turbo coding: Turbo coding is the modern development in the area of forward error correction codes that can achieve near Shannon-limit performance. Maximum theoretical bounds on turbo codes are based on union bound. The basic idea of turbo codes involves usage of two identical recursive systematic convolutional (RSC) codes in parallel with some kind of interleaving in between. Data is configured in finite blocks corresponding to the interleaver size. At the decoder end there are two soft input-output component decoders. Decoding here is an iterative process. Probabilistic information is exchanged between decoders. They use Log Likelihood Ratios (LLR) to compute extrinsic information. As Suboptimal Max-Log-Map algorithm with Extrinsic Scaling Factor (ESF) used for decoding results in performance loss below $0.2 \mathrm{~dB}$ thus, when this algorithm is combined with turbo coding then it eliminates requirement of knowledge about SNR.

Link Adaptation: It is defined as collection of techniques for varying and adjusting the transmission parameters. It results in a system that responds in a better way to the dynamic nature of communication channel. On the basis of radio link quality, link adaptation enables eNodeB to adjust transmitter power of UE (User Equipment) and throughput. It is typically based on selection of modulation scheme and code rate known as Adaptive Modulation and Coding (AMC). UE transmits a channel quality indicator (CQI) as a feedback on the basis of which eNodeB selects appropriate modulation and coding scheme (MCS). Feedback CQI can be periodic as well as aperiodic.

MIMO (Multiple Input Multiple Output): In order to meet ambitious requirements of LTE, MIMO technology is deployed in this standard. Different MIMO algorithms are used in LTE which can be categorised as spatial multiplexing, beam-forming, transmit diversity and receive diversity. The degree of improvement of data rates is linearly proportional to number of transmitter antennas. Number of transmit antennas vary in downlink and uplink transmission. LTE standard provides downlink configurations of up-to 4 transmit antennas. For LTEAdvanced downlink transmission can accommodate 8transmit antennas. Mostly 2-transmit antennas are used for uplink. In order to maximize gains in throughput, flexibility is the requirement in MIMO. Different transmission modes are available in LTE regarding MIMO technology. These modes are outlined to take the best advantage of distinct channel and multipath conditions. Mobility, SNR and channel correlation are the factors governing efficient selection of the best MIMO mode. Here low correlation indicates signal orthogonality.

OFDM (Orthogonal Frequency Division Multiplexing): To fulfil the requirement of physical layer (higher data rates, flexible bandwidth etc), LTE design has included OFDM as basis for downlink physical layer. Multiple narrow band sub-carriers which are mutually orthogonal in the frequency domain are spread over wide-bandwidth. Thus it simply converts wideband frequency selective channels into a set of differing flat fading sub-channels. It has ability to mitigate inter-symbol interference. Fast Fourier Transform (FFT) and Inverse FFT (IFFT) are applied to retrieve data stream and modulating as well as combining data symbols respectively. Size of FFT varies according to the bandwidth used.

Single Career Frequency Division Multiplexing (SCFDMA) is deployed for uplink in LTE and is a variant of OFDM scheme. It is an intelligent technique that smartly combines low Peak to Average Power Ratio (PAPR) of single carrier systems with multi-path resistance subcarrier frequency allocation done by OFDM.

Thus an overview about four key technologies of LTE clarifies their importance in this wireless system. Their combination with LTE has enhanced system parameters greatly. Next section is literature survey regarding involved technologies of LTE. Further in section 4 issues and challenges are found, followed by open issues and future scope in section 5 . Then conclusion has been given in section 6 .

\section{LITERATURE SURVEY}

Turbo Coding: Main parameters of turbo coding,affecting QoS of this particular wireless system, mainly include type of interleaver, block length, downlink rates and number of iterations.

Patel SnehaBhanubhai in [8]explained about encoder and decoder of turbo coding including interleaving criteria. BER had been calculated for different values of $\mathrm{E}_{\mathrm{b}} / \mathrm{N}_{\mathrm{o}}$ for distinct sets of block lengths like 512, 1024 and 2048. Now, it can be said that as the block length increases, decoding performance also improves. Moreover for larger 
block lengths, BER is less for a given value of $E_{b} / N_{o}$. In short, smaller blocks are restrictive for better outcome. In turbo coding interleaver block lengths that are supported in LTE is at the maximum 6144 bits. Transport block exceeding this size are segmented into smaller codeblocks. Considering the downlink rates, generally $1 / 3$ is used.

AjitNimbalker in [7]proposed the problem that existing turbo interleavers are unable to meet requirements of high throughput of system. So to rectify this problem Almost Regular Permutation (ARP) and Quadratic Polynomial Permutation (QPP) interleavers are recommended. A major advantage of the ARP design is that it is vectorizable with respect to different parallelism factors. On the other hand, QPP interleavers are maximum contention-free and maximum vectorizable. LTE turbo codes can hold input block sizes between 40 and 6144bits. Upper limit ensures that IP packet has been segmented into maximum of 2 code blocks. Thus requirement is of limited set of interleaver sizes suitable distributed between $\mathrm{K}_{\min }=40$ and $\mathrm{K}_{\max }=6144$. Interleavers are defined such that decoders can be put into practice with various parallelism factors $\mathrm{M}$. For a given block size $\mathrm{K}$ very smaller window sizes can deteriorate the decoder performance. Simulations represent that the minimum window size that can be allowed without consequential performance loss is $\mathrm{W}_{\min }=$ 32 for practical block size of 2000 to 6000 bits [2]. Parallelism for each data packet is fine tuned in LTE according to required output of respective factors.

TimoLehnigk-Emden in [5]represented that number of iterations degrades the peak throughput, latency and affects energy consumption of system. Increased number of iterations bounds system performance. There is a drastic reduction in BER and BLER (Block Error Rate) with increase in number of iterations. As the iterations increases from 1 to 7, desired BER can be easily obtained [10]. Thus no doubt with increase in iterations performance becomes superior but gain also decreases. So, fixed number of iterations for every system is not preferred. Different iteration-control mechanisms are used to correctly detect the decoded blocks without sacrificing communication performance. For example re-encoding approach saves up-to $80 \%$ of iterations. Advanced iteration control techniques can significantly reduce number of iterations and enhances system performance. Via approaches like turbo synchronization, performance gain of $0.25 \mathrm{~dB}$ can be achieved.

Similarly, Peng Zhu in [10]explained different decoding mechanisms and evaluated code performance under distinct AWGN channel parameters. It can be concluded that in order to get better performance from turbo codes, for a particular value of SNR, more number of iterations as well as longer information sequence will result in excellent decoding algorithm. Thus longer frame lengths are required. But at the same time frame length will determine time delays of transmission and decoding. So there remains trade-offs.
MIMO: There are different aspects of MIMO that bounds the performance in LTE. SNR and then BER play a very important role here.Selection of appropriate MIMO technique is equally crucial.

Sunil Joshi in [4] discussed about two vital aspects of MIMO i.e. Spatial Multiplexing (SM) and Transmit Diversity (TD). Their effect on system performance has been given considering affect of rich and poor scattering channel conditions. Presence of rich scattering environment strengthens system performance and poor scattering environment degrades system throughput and limits its performance. Depending on signal strength SM or TD are applied. High SNR is the requirement for spatial multiplexing and for low SNR diversity set up can be used. For high-speed users Transmit Diversity is preferable than SM.

Rician factor expresses the relative strengths of direct and scattered component. It has shown that in case of SM for SNR below $13 \mathrm{~dB}$, throughput of system is decided by Rician factor K. As $\mathrm{K}$ changes from 0 to 1 there is a linear increase in throughput. On the contrary in case of TD, multipath do not affect throughput. It remained constant above $6 \mathrm{~dB}$.

Moreover mobility factor is also vital. As the speed changes from $20 \mathrm{Km} / \mathrm{Hr}$ to $60 \mathrm{Km} / \mathrm{Hr}$, there is a decrease in $50 \%$ of throughput for SM. Next capacity increases by $17 \%$ for LOS in SM. For poor scattering environment capacity is $20 \%$ lower than rich scattering scenario in SM. same is the case for TD. LOS enhances up-to $22 \%$ and for NLOS capacity for poor scattering is $14 \%$ lower than richer case. Thus, presence or absence of rich or poor scattering affects throughput bounds of system performance greatly.

Talking about Pre-coding, it modifies layer signals before transmission. Its choices are defined in a lookup table called as code-book. Only layer-two case employs spatial multiplexing. Pre-coding with single layer is limited to a $0^{\circ}, \pm 90^{\circ}$ or $180^{\circ}$ phase-shift. Amongst open loop and closed loop spatial multiplexing, greatest potential gains in throughput come from closed loop technique up-to peak rates. Closed loop spatial multiplexing after receiving detailed information from UE is able to modify pre-coding matrix and modulation scheme to the actual conditions of UE. This allows throughput to attain theoretical limits that are set by multipath conditions and SNR [2].

Stefan Schwarz in [9]proposed several increasingly tight and confined performance bounds on OFDM based MIMO systems in LTE. It considered channel capacity as ultimate bound on throughput. Analysis has shown that large part of performance deterioration is because of system overhead necessary for timing and frequency synchronization as well as channel estimation, finite code block length etc. So many bounds are present in system depending on prevailing conditions. These bounds are Open Loop Mutual Information (OLMI) bound, Closed Loop Mutual Information (CLMI) bound, WidebandCLMI with linear receiver bound, Bit Interleaving Coded 
Modulation (BICM) bound, shifted or quantized BICM bound. All these bounds greatly reduce the optimum throughput of LTE system. Loss in performance is high for larger antenna configurations. These flaws are required to be removed for better communication systems. Paper had compared observed bounds to the performance of a practical LTE system. It concluded that LTE achieves around 50\% channel capacity that too with the assumption of perfect channel estimation as well as frequency synchronization.

OFDMA AND SC-FDMA:Choice of bandwidth is very important here. It affects number of accommodated carriers which in turn will affect symbol length etc. Upper limit of sub-carriers is 2048 with $15 \mathrm{KHz}$ spacing. A mobile must have capability to deal with all 2048 of them but the base station requirement is to support up-to 72 subcarriers only. Further higher modulation schemes like 64QAM, 16QAM or simply QPSK can be selected within OFDM signal according to prevailing conditions.

Luis Angel Maestro Ruiz de Temino in [2] proposedperformance evaluation of SU-MIMO for both OFDMA and SC-FDMA. It has been shown that OFDMA performs better than SC-FDMA for 2-receive antenna configuration especially in the case where spatial multiplexing is used with a gain of $3 \mathrm{~dB}$ at high SNR. But the gap of $1 \mathrm{~dB}$ disappears when antenna number increased up-to 4. Moreover, spatial processing of Space Frequency Coding (SFC) results in Peak to Average Power Ratio (PAPR) increase for SC-FDMA up-to 0.6dB. But the gap between different schemes like spatial multiplexing, space frequency coding, SIMO etc highly reduces when configuration includes 4 receiver antennas.

Mark Geles in [3] represented analysis of performance of optimal decoder for SC-FDMA. Considering correlated and unrelated Rayleigh fading channels in low and high SNR regimes, BER closed form bounds have been presented. It also demonstrated that value of BER for which there is a change in slope corresponds to size of DFT. Further it represents that for smaller DFT size flaring behaviour appears. Thus behaviour of system varies depending upon SNR and hence DFT size.

ISI may affect SC-FDMA signal in case of multipath. It makes frequency domain equalization sub-optimal. For most of the cases optimal ML detection is prohibitively complex. This makes it advantageous mostly as performance bound for sub-optimal detection schemes. Employing MLD for QPSK with large DFT sizes is impractical. It is because of very high computational complexity. For larger DFTs BPSK is preferred. Larger the DFT size, smaller is the BER value. Thus parameters like DFT size, Modulation and coding scheme and detection algorithm etc are interrelated. Hence a performance bound always remains.

Link adaptation:SNR and SINR (Signal to Interference and Noise Ratio) are two parameters that helps the system to select appropriate CQI and then in turn an efficient MCS for transmissions.

Kari Aho in [1]proposed that channel quality measurement is very necessary, because on its basis appropriate modulation and coding scheme is chosen. The goal of link adaptation is to attain desired BLER operating point. But problem arises when biased or non-consistent CQI is reported resulting in compromised operation point and hence system performance. It may be due to hardware inaccuracy, misconfiguration or error in calibration. Nonconsistent CQI reporting by UE affects system performance depending on amount of data/packets. If bias is relatively high then only biased CQI values will affect performance of system. For link setups of longer duration system has enough time to correct the bias. Thus, bias is a boundation mainly for systems communicating for shorter durations.

Performance of fixed and random bias is shown. It concluded that non-aggressive bias can even improve parameters as $90 \%$ user throughput. On the contrary aggressive bias caused $10-15 \%$ loss for UEs. It has been further shown that outer loop link adaptation has ability to correct $\pm 2 \mathrm{~dB}$ bias range without varying spectral efficiency and user throughput.

V.A.Loshakov in [6] investigated downlink performance of LTE physical layer on MIMO antenna with CQI feedback. Bounds have been searched for different channel models. Based on varying factors throughput comparison has been done. It further differentiated distinct CQIs and has shown that CQI8 has the lowest BLER than CQI 11. This is because latter requires higher SNR to achieve same level of BLER as that of former one. It shows that for 64QAM (CQI 11) that has better throughput requires higher SNR to achieve a good level of BLER. CQI values are used to choose optimum resource block. One of the methods of choosing accurate CQI is based on a set of BLER thresholds. The UE would report CQI with respect to MCS which ensures BLER $\leq 10 \%$.

For high SNR, higher order modulation schemes having higher spectral efficiency are utilized (64-QAM). Oppositely, for low SNR values, modulation scheme with lower order like QPSK is preferred. It is more robust against transmission errors but having low spectral efficiency. Moreover, better the channel quality, higher will be used code rate. Value of CQI, on the basis of which efficient modulation scheme and hence code rates are chosen also depends on receivers. The receivers with better front end or more powerful signal processing algorithms delivers a higher CQI.

\section{ISSUES AND CHALLENGES}

Different issues are related to different key technologies. On the basis of literature survey done in previous section issues and challenges are represented as follows in a tabular form: 
Table 2: Issues and Challenges of four enabling technologies

\begin{tabular}{|c|c|c|}
\hline Paper & Issues & Challenges \\
\hline Kari Aho [1] & $\begin{array}{l}\text { Biased or non- } \\
\text { consistent channel } \\
\text { quality indicator may } \\
\text { arise due to issues of } \\
\text { hardware inaccuracy, } \\
\text { error in calibration etc. }\end{array}$ & $\begin{array}{l}\text { It is challenging to select } \\
\text { how tightly BLER } \\
\text { operation point must be } \\
\text { there so as to shun any } \\
\text { compromise in system } \\
\text { performance. }\end{array}$ \\
\hline $\begin{array}{l}\text { AjitNimbalke } \\
\text { r [7] }\end{array}$ & $\begin{array}{l}\text { There is a severe link- } \\
\text { level performance loss } \\
\text { with WCDMA turbo } \\
\text { interleavers for higher } \\
\text { code rates. } \\
\text { Poor minimum } \\
\text { distance at many block } \\
\text { sizes in interleaver and } \\
\text { puncturing are two } \\
\text { main issues resulting } \\
\text { in deterioration of } \\
\text { performance } \\
\text { WCDMA of } \\
\text { interleavers. }\end{array}$ & $\begin{array}{l}\text { Embedding } 3 \text { key } \\
\text { parameters i.e. flexible } \\
\text { parallelism, efficient } \\
\text { memory organisation } \\
\text { and effective support for } \\
\text { Radix-4 decoding in } \\
\text { turbo interleavers is a } \\
\text { big challenge. }\end{array}$ \\
\hline $\begin{array}{l}\text { Luis } A^{\prime} \text { ngel } \\
\text { Maestro Ruiz } \\
\text { de Temiño } \\
{[2]}\end{array}$ & $\begin{array}{l}\text { Tight synchronization } \\
\text { between users is } \\
\text { required for FFT } \\
\text { reception, complexity } \\
\text { in adaptive sub-carrier } \\
\text { assignment, Suffers } \\
\text { from Noise } \\
\text { enhancement and } \\
\text { Poor peak to average } \\
\text { power (PAPR) ratio is } \\
\text { particularly as issue } \\
\text { for uplink. }\end{array}$ & $\begin{array}{l}\text { Modelling of non-linear } \\
\text { receivers that can fight } \\
\text { against issues of FDMA } \\
\text { is still quiet challenging. } \\
\text { Because of very high } \\
\text { computational } \\
\text { complexities involved, it } \\
\text { becomes more } \\
\text { challenging to } \\
\text { implement respective } \\
\text { algorithms. }\end{array}$ \\
\hline $\begin{array}{l}\text { Stefan } \\
\text { Schwarz [9] }\end{array}$ & $\begin{array}{l}\text { Performance } \\
\text { degradation occurs due } \\
\text { to varying overheads } \\
\text { involved. } \\
\text { Moreover, accurate } \\
\text { channel state } \\
\text { information prediction } \\
\text { is very difficult due to } \\
\text { different obstructions } \\
\text { in its path. } \\
\text { Undesirable delay in } \\
\text { feedback as well as } \\
\text { time variability is also } \\
\text { present. }\end{array}$ & $\begin{array}{l}\text { Role of receiver is very } \\
\text { crucial in MIMO. It is } \\
\text { difficult to design } \\
\text { receivers with efficient } \\
\text { front-ends which can } \\
\text { effectively decode signal } \\
\text { and hence accurate CSI } \\
\text { can be provided. Thus } \\
\text { CSI makes MIMO more } \\
\text { challenging. }\end{array}$ \\
\hline $\begin{array}{ll}\text { Mark } & \text { Geles } \\
{[3]} & \end{array}$ & $\begin{array}{l}\text { Because of multipath, } \\
\text { SC-FDMA r may } \\
\text { appear at receiver } \\
\text { troubled by Inter } \\
\text { Symbol Interference } \\
\text { (ISI). Moreover for } \\
\text { sub-optimal detection } \\
\text { of SC-FDMA error } \\
\text { rate flaring behaviour } \\
\text { is present. }\end{array}$ & $\begin{array}{l}\text { It is challenging to } \\
\text { implement an optimal } \\
\text { detection scheme due to } \\
\text { respective performance } \\
\text { bounds. }\end{array}$ \\
\hline $\begin{array}{l}\text { TimoLehnigk } \\
\text {-Emden [5] }\end{array}$ & $\begin{array}{l}\text { At the receiver end, } \\
\text { turbo decoder } \\
\text { consumes maximum } \\
\text { time to generate } \\
\text { output. As a result } \\
\text { delay increases. }\end{array}$ & $\begin{array}{l}\text { It is very difficult to } \\
\text { reduce complexity of } \\
\text { turbo decoder with } \\
\text { increased number of } \\
\text { iterations. There remains } \\
\text { trade-off retween } \\
\text { iterations, complexity } \\
\text { and hence computation } \\
\text { time. }\end{array}$ \\
\hline
\end{tabular}

V.

\section{OPEN ISSUES AND FUTURE SCOPE}

Many questions are still left unanswered regarding technologies implemented in LTE. Talking about turbo coding the exact procedure with which information can be passed to next decoder as well as next iteration stage is an issue of research. Work is still being researched for improved design guidelines and to link interleaver specification to code performance. Moreover, SU-MIMO has not been standardized in release8 .With acquisition of MIMO technology in satellite LTE air interface there remains impact of Round Trip Propagation Delay (RTPD). Misalignment occurs between reported CQI at eNodeB and current CQI at UE due to long RTPD when report is sent via Geo satellite. In order to make SC-FDMA perform as satisfactory as OFDMA, future work involves investigation of advanced non-linear receivers based on turbo equalization to boost SC-FDMA performance. Talking about future scope, 4G LTE is leading to new innovations and transforming mobile lives. It promises to revolutionize the use of data services on the move. It has ability to entirely transform mobile ecosystem. With the support of four enabling technologies, LTE provides constant sound connectivity with incredible reach to refine the user experience.

\section{CONCLUSION}

The objective of this paper was to represent the performance bounds in terms of QoS parameters of different techniques in LTE. It can be concluded that accurate calculation of SNR is very difficult using any algorithm in all technologies mentioned in this paper. Apart from that different technologies are having different limitations in their performance. Turbo coding has its bounds mainly with respect to block length, iterations and decoding algorithms. Link adaptation has limited number of modulation and coding schemes. In case of MIMO different factors implies bounds on throughput, peak capacity and for its pre-coding. For OFDM FFT size and peak average power ratio bounds its level of performance. Although performance bounds are there but still amalgamation of Turbo codes, Link Adaptation, MIMO and OFDM in LTE is working up to the mark as well as giving tough competition to existing technologies.

\section{REFERENCES}

[1] Aho Kari, Alanen Olli, KaikkonenJorma (2011). "CQI Reporting Imperfections and their Consequences in LTE Networks" The Tenth International Conference on Networks (ICN 2011).

[2] Angel Maestro Ruiz de Temin Luis, Berardinelli Gilberto, Frattasi Simone, Pajukoski Kari and MogensenPreben (2009). "Single-User MIMO for LTE-A Uplink: Performance Evaluation of OFDMA vs. SC-FDMA" Radio and Wireless Symposium, 2009, RWS'09 IEEE.

[3] Geles Mark, Averbuch Amir, AmraniOfer and EzriDoron (2012). "Performance Bounds for Maximum Likelihood Detection of Single Carrier FDMA" Communications, IEEE Transactions on(Volume: 60, Issue: 7).

[4] Joshi Sunil, Gupta Deepak, Bapna P.C., Kothari Neha, SutharRashmi (2011). "Throughput performance of $2 \times 2$ MIMO LTE downlink in a spatial correlation based microcellular channel for wireless broadband networks" International Journal of Wireless \& Mobile Networks (IJWMN) Vol. 3, No. 5.

[5] Lehnigk-Emden Timo, WasenmüullerUwe, Gimmler Christina, and Wehn Norbert (2009). "Analysis of Iteration Control for Turbo Decoders in Turbo Synchronization Applications" Manuscript 
prepared for Adv. Radio Sci.with version 2.3 of the LATEX class copernicus.cls.

[6] Loshakov V.A., Al-Janabi H.D., Al-Zayadi H.K. (2012). “Adaptive control signal parameters in LTE technology with MIMO" Electronic scientific specialized edition Journal Telecommunications Problems.

[7] NimbalkerAjit, Blankenship Yufei, Classon Brian (2008). "ARP and QPP Interleavers for LTE Turbo Coding" in WCNC.

[8] Patel SnehaBhanubhai, Grace Mary Shajan, Dalal D. Upena (2012). "Performance of Turbo Encoder and Turbo Decoder for LTE" International Journal of Engineering and Innovative Technology (IJEIT) Volume 2, Issue 6.

[9] Schwarz Stefan, Simko Michal and Rupp Markus. "On performance bounds for MIMO OFDM based wireless communication Systems" A1 Telekom Austria AG and the Institute of Telecommunications, Vienna University of Technology.

[10] Zhu Peng, Zhu Jun, Liu Xiang (2013). "A Study on Turbo Code Performance Based on AWGN Channel" Proceedings of the 2nd International Conference on Computer Science and Electronics Engineering (ICCSEE 2013).

[11] Ian F. Akyildiz, David M. Gutierrez-Estevez, Elias Chavarria Reyes (2010). "The evolution to 4G cellular systems: LTEAdvanced"Physical Communication 3 (2010) 217-244. 